\title{
Bilateral Internal Auditory Canal (IAC) \\ Duplication with Congenital Sensorineural Hearing Loss (SNHL)-A Case Report
}

\author{
Nur Saadah M. ${ }^{a}$, Nik Adilah N. O. ${ }^{a}$, Ahmad Aizuddin M. J. ${ }^{b}$, Mohd Shafie A. ${ }^{b}$, Mohd Khairi M. D. ${ }^{a}$ \\ ${ }^{a}$ Department of Otorhinolaryngology-Head \& Neck Surgery, School of Medical Sciences, Universiti Sains Malaysia \\ ${ }^{b}$ Department of Radiology, School of Medical Sciences, Universiti Sains Malaysia
}

\author{
Keywords \\ Duplicate IAC, Bilateral Duplicate IAC, \\ sensorineural hearing loss, Down \\ syndrome \\ Corresponding Author \\ Nik Adilah Nik Othman \\ Department of Otorhinolaryngology-Head \\ \& Neck Surgery, School of Medical \\ Sciences, Universiti Sains Malaysia, 16150, \\ Kubang Kerian, Kelantan, Malaysia. \\ Tel No: +609-7676428 \\ E-mail: adilahkk@usm.my \\ Received: 17 January 2021; Accepted: 9 \\ February 2021 \\ Doi: https://doi.org/10.31436/imjm.v20i4
}

\begin{abstract}
Duplication of the internal auditory canal is a rare entity. It can be unilateral or bilateral involvement and usually found during an investigation of sensorineural hearing loss or facial nerve palsy. We report this image finding in a child with Down syndrome with bilateral profound sensorineural hearing loss. There was no facial nerve palsy. We highlight the rarity of this image finding and the importance of imaging for the detection of middle and inner ear abnormality in a case of congenital sensorineural hearing loss as it helps in decision making for the suitable treatment.
\end{abstract}

\section{INTRODUCTION}

The internal auditory canal (IAC) is a structure that transmits facial nerve and vestibulocochlear nerve (VCN) from the brainstem to the inner ear. Its embryological development was influenced by the trophic effect of the VCN. ${ }^{1}$ Therefore, there is an association between hypoplasia of the nerve with the IAC abnormality and sensorineural hearing loss (SNHL). This anatomical anomaly is rare and only about eight cases involving the bilateral duplication IAC have been reported. ${ }^{2}$ Middle and external ear anomalies are well described in Down syndrome (DS) while inner ear anomalies are less recognized. DS is a genetic disorder affecting 1 in every 800 live births and associated with multiple health conditions including hearing loss. $80 \%$ of the hearing loss in DS is due to the conductive type (CHL) and only 4-20\% are due to mixed (MHL) and SNHL. 3,4

\section{CASE HISTORY}

A four-year-old Malay boy with underlying Down Syndrome and has congenital heart disease, global developmental delay, and congenital cataract bilaterally. He was referred to the ORL clinic for speech delay as there was no meaningful word utterance yet. Birth history was uneventful with no history of prolong ototoxic antibiotics used, severe jaundice, high-grade fever, meningitis, head trauma, ear infection, or even family history with congenital hearing problem. On examination, both pinna were normal and symmetry but bilateral ear canals were narrow, with no sign of infection such as inflammation and pus discharge in the ear canals. Due to the narrow canals, both the tympanic membranes were not well visualized.

The boy was referred to the audiologist and the auditory brainstem response showed absence of wave $\mathrm{V}$ at 100 $\mathrm{dB}$ intensity in both ears suggestive of bilateral profound hearing loss. He was considered as a candidate for cochlear implant and High-Resolution Computed Tomography (HRCT) of the temporal bone and Magnetic Resonance Image (MRI) of Internal Auditory Meatus was requested to assess the anatomical bony structures of the middle and inner ear and the IAC neural contents respectively. 
HRCT temporal bone revealed a duplication of the IAC in both ears with a complete bony septum on the right side dividing the facial canal and vestibulocochlear canal along with the IAC while the left side showed an incomplete bony septum (Figure 1). MRI showed normal course and caliber of both right and left facial nerves at the superior part of IAC. The right VCN located at the inferior part of IAC was smaller in caliber which is only $0.6 \mathrm{~mm}$ in diameter (compared to the normal $1.2 \mathrm{~mm}$ diameter of the left $\mathrm{VCN}$ ) and was visualized from pons and through the IAC (Figure 4).

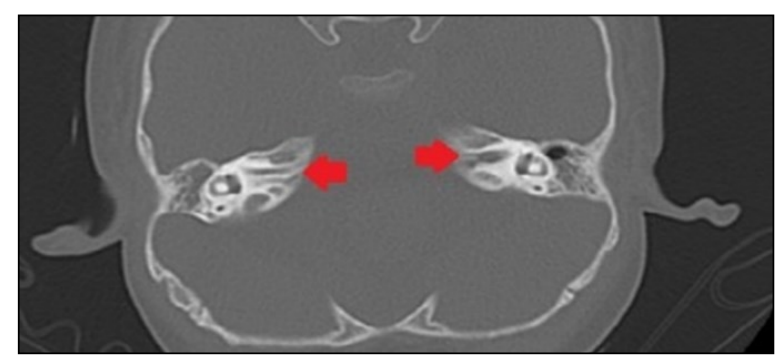

Figure 1: An axial view HRCT Temporal bone showing duplication of bilateral IAC (red arrows)

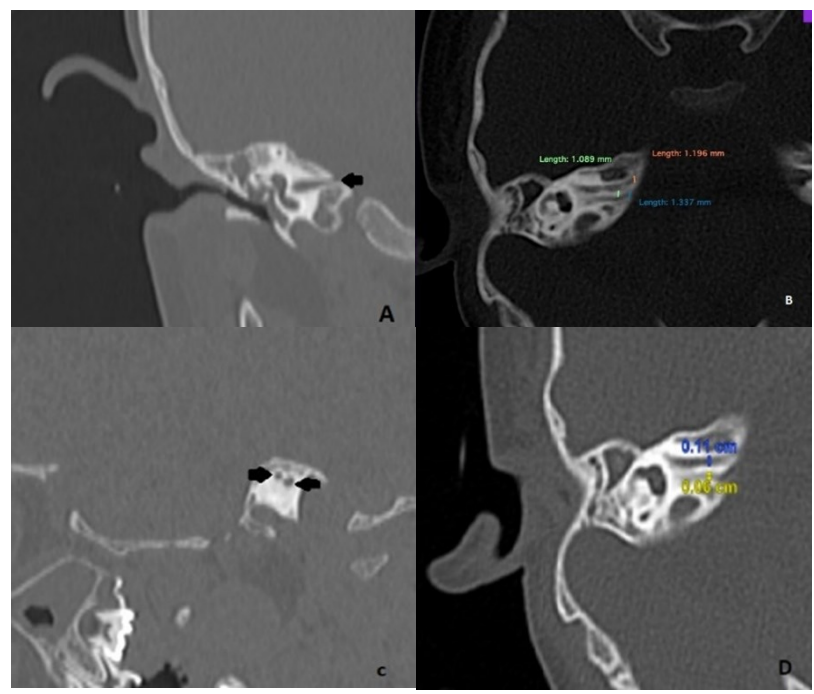

Figure 2: Reconstructed image HRCT Temporal bone following the right IAC (coronal view) (A). Complete duplication of the IAC with two partitions anteriorly measuring $1.196 \mathrm{~mm}$ and posteriorly measuring $1.337 \mathrm{~mm}$ (at porous acousticus) with the bony septum in between measuring $1.08 \mathrm{~mm}$ (axial view) (B). In the sagittal view, there is an anterosuperior and posteroinferior canal in the right internal auditory meatus (black arrows) (C). The diameter of a duplicate canal with a vestibulocochlear canal measuring $0.6 \mathrm{~mm}$ in comparison to a facial canal $1.1 \mathrm{~mm}$ (axial view) (D).

\section{DISCUSSION}

Narrowing of IAC constitutes about $12 \%$ of the temporal bone anomaly. ${ }^{5}$ There are two main theories regarding the pathogenesis of narrow IAC. Firstly, it is due to the trophic effect of the embryonic cochlear and vestibule on the VCN. Atrophy or hypoplasia of the VCN will affect the chondrification and ossification of mesoderm surrounding the nerves for the development of the IAC and later resulting in a narrow IAC. A second theory is due to the stenosis of the IAC that obstructs the growth and development of the VCN and causing nerve hypoplasia. However, the second theory is not widely accepted due to the presence of normal morphology and function of the facial nerve inside the stenotic IAC. ${ }^{5-7}$ This is evidenced in most of the cases, including this case.

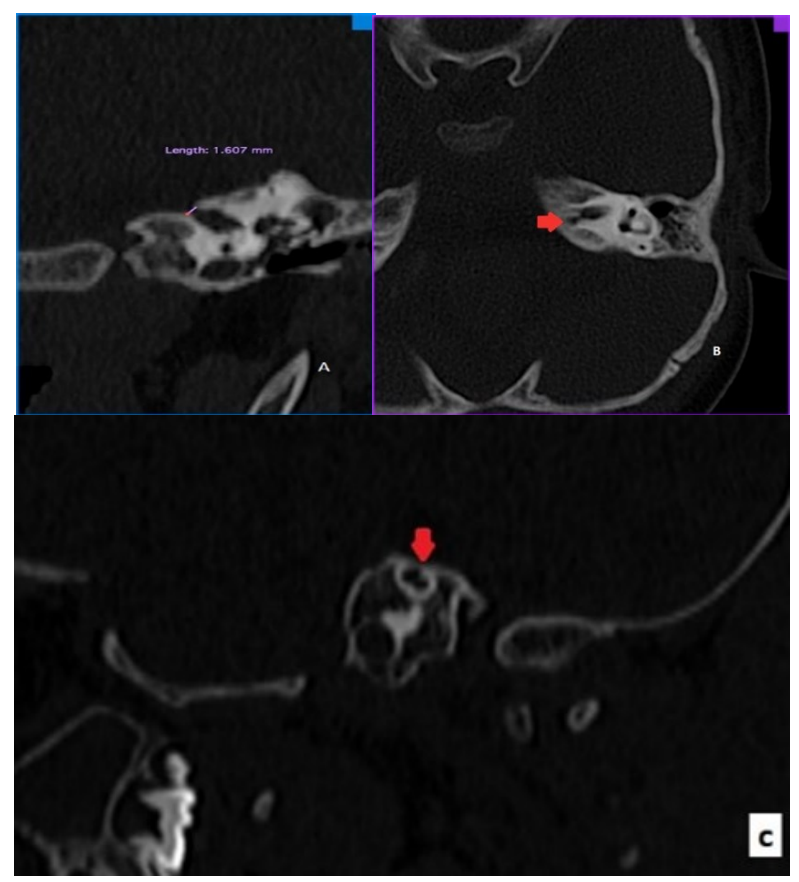

Figure 3: Reconstructed images HRCT Temporal bone following left IAC shows narrowing left porus acousticus measuring $1.607 \mathrm{~mm}$ (coronal view) (A). There is incomplete duplication of the IAC (axial view) (B). There were anterosuperior and posteroinferior partition seen inside (sagittal view) (C)

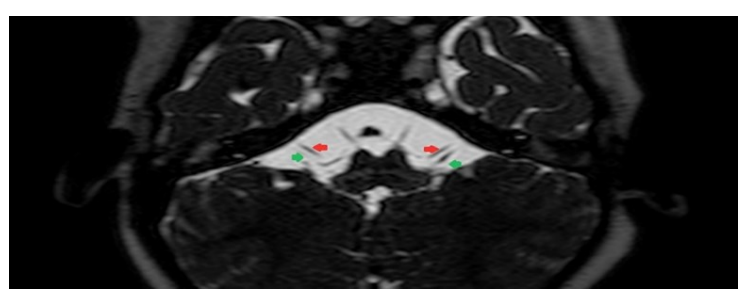

Figure 4: T2 weighted image (axial) of MRI IAC showing the intracranial course of the facial nerve (red arrows) and VCN (green arrow) from the pons toward IAC. The right VCN is thinner compared to the left side, and it disappeared upon entering IAC.

Duplication of the IAC by a bony septum can be complete and incomplete. This causes the narrowing of the canals in the IAC. The narrowing of IAC can be 
due to congenital or acquired causes such as Paget's disease, fibrous dysplasia, or osteoporosis. It is also associated with the findings of abnormal structure of the inner ear such as labyrinthine aplasia, dysplasia of semicircular canal and vestibule, and incomplete partition of the cochlea. Furthermore, it also can be associated with a systemic syndrome such as Goldenhar syndrome, Treacher Collin syndrome 5 , and CHARGE syndrome $^{7}$ but from our extensive literature review, there is no case reported in Down Syndrome.

Previously, cochlear implant (CI) was not performed in cases of narrow IAC as it is believed that there is a possibility of the absence of the VCN. The presence of normal VCN is important for the good outcome of the auditory stimulation post-implantation. Nowadays, the advancement of CT and MRI scans also changes the perception of preoperative assessment of patients' candidacy for cochlear implantation. Evaluation of the bony structure of IAC is best measured using HRCT of Temporal bone with the thickness of $1 \mathrm{~mm}$ and capability of multiplanar reconstruction (MPR) in the bone window. At our center, we are using CT Machine Toshiba Aquilion Prime 160 slices. In our case, the right IAC is narrow with a complete bony septum in between the two canals, measuring $1.1 \mathrm{~mm}$ anteriorly and $1.3 \mathrm{~mm}$ posteriorly at porous acousticus level (Figure 2, B). On the left side, the porous acousticus caliber is narrow measuring $1.6 \mathrm{~mm}$ with the incomplete bony septum (Figure 3, B). Furthermore, like other DS patients, our patient also has bilateral ear canal stenosis. Canal stenosis is defined as a canal diameter less than $4 \mathrm{~mm} .{ }^{8}$ There is no direct relation between the narrow IAC and EAC as these two structures have different origins embryologically.

The assessment of the facial nerve and VCN pathways was done using Magnetic Resonance Image (MRI) machine Philip Achieva 3Tesla with T2DRIVE $1 \mathrm{~mm}$ acquisition. The MRI finding, in this case, showed hypoplastic right VCN in comparison to the ipsilateral facial nerve while on the left side, the calibers of both nerves were normal in the IAC. According to the first theory stated above, hypoplasia of the right VCN caused ossification of its surrounding mesoderm suggesting why the right IAC is having complete duplication in comparison to incomplete duplication on the left side.
There is a high prevalence of hearing loss in children with DS as much as 38\%.9 The most common type of hearing loss in DS is a conductive type which is caused by cerumen impaction and middle ear pathologies, such as middle ear effusion, acute otitis media, and eardrum perforations. SNHL is uncommon in children with DS with a prevalence of $4.5 \%$ and about $40 \%$ was found to be congenital in origin. ${ }^{4}$ For the management of hearing loss in DS, it depends on the type either conductive or sensorineural. Ventilation tube insertion has been shown to improve the hearing acuity in CHL while in SNHL, the treatment for mild to moderate severity is the hearing aid and for severe to profound hearing loss is CI. Previously it is only performed on a candidate with no disability. However, a CI experience in a child with a disability in UK and Ireland showed a positive impact on their learning and communication though at a slower pace than a child with no disability. ${ }^{3}$ As in our case, the child may benefit from a CI on the left ear as both the middle and inner ear structures were normal. However, CI cannot replace other types of communication for these children, as the cause of speech delay may be multifactorial, and it is important to give parents realistic expectations prior to surgery.

\section{CONCLUSION}

Narrowing and duplication of IAC are detected by imaging upon an investigation of SNHL. Meticulous pre-implantation investigations should be done due to the possibility of temporal bone abnormality that can be encountered during surgery and assessing the neuroanatomy of the cochlear nerve. A child with DS with severe to profound SNHL can benefit from the CI.

\section{CONFLICT OF INTEREST}

None

\section{ACKNOWLEDGEMENT}

We would like to express our gratitude to the patient's parents that gave their permission for this case report. 


\section{REFERENCES}

1. Jackler RK, Luxfor WM, House WF. Congenital malformations of the inner ear: a classification based on embryogenesis. The Laryngoscope. 1987; 97:2-14.

2. Manchanda S, Bhalla AS, Kumar R, Kairo AK. Duplication Anomalies of the Internal Auditory Canal: Varied Spectrum. Indian Journal of Otolaryngology and Head \& Neck Surgery. 2017:15.

3. Hans P, England R, Prowse S, Young E, Sheehan P. UK and Ireland experience of cochlear implants in children with Down syndrome. International journal of pediatric otorhinolaryngology. 2010; 74:260-4.

4. De Schrijver L, Topsakal V, Wojciechowski M, Van de Heyning P, Boudewyns A. Prevalence and etiology of sensorineural hearing loss in children with down syndrome: A cross-sectional study. International journal of pediatric otorhinolaryngology. 2019; 116:168-72.

5. Kew TY, Abdullah A. Duplicate internal auditory canals with facial and vestibulocochlear nerve dysfunction. The Journal of Laryngology \& Otology. 2012; 126:66-71.

6. Yates JA, Patel PC, Millman B, Gibson WS. Isolated congenital internal auditory canal atresia with normal facial nerve function. International journal of pediatric otorhinolaryngology. 1997; 41:1-8.

7. Weon YC, Kim JH, Choi SK, Koo J-W. Bilateral duplication of the internal auditory canal. Pediatric radiology. 2007; 37:1047-9.

8. Dy AES, Lapeña Jr JFF. External auditory canal dimensions, age, and cerumen retention or impaction in persons with Down syndrome. Annals of Otology, Rhinology \& Laryngology. 2018; 127:253-7.

9. Nightengale E. Hearing Loss in Children with Down Syndrome. The Hearing Journal. 2018; 71:10-2. 